\title{
Generalized fractional inequalities for quasi-convex functions
}

\author{
S. Ullah' ${ }^{1}$, G. Farid ${ }^{2}$, K.A. Khan ${ }^{3}$, A. Waheed ${ }^{2}$ and S. Mehmood ${ }^{4 *}$
}

"Correspondence:

smjg227@gmail.com

${ }^{4}$ GBPS Sherani, Hazro Attock, Pakistan

Full list of author information is available at the end of the article

\begin{abstract}
The class of quasi-convex functions contain all those finite convex functions which are defined on finite closed intervals of real line. The aim of this paper is to establish the bounds of the sum of left and right fractional integral operators using quasi-convex functions. An identity is formulated which is used to find Hadamard-type inequalities for quasi-convex functions. Connections with some known results are analyzed. Furthermore, some implications are derived by considering some examples of quasi-convex functions.
\end{abstract}

MSC: 26D15; 26A51; 32F99; 41A17; 33E12

Keywords: Convex function; Quasi-convex function; Hadamard inequality; Mittag-Leffler function; Fractional integral operators

\section{Introduction}

Definition 1 Let $I$ be an interval of real numbers. Then a function $f: I \rightarrow \mathbb{R}$ is said to be convex function, if for all $a, b \in I$ and $0 \leq t \leq 1$ the following inequality holds:

$$
f(t a+(1-t) b) \leq t f(a)+(1-t) f(b) .
$$

A convex function is interpreted very nicely in the coordinate plane by the Hadamard inequality

$$
f\left(\frac{a+b}{2}\right) \leq \frac{1}{b-a} \int_{a}^{b} f(x) d x \leq \frac{f(a)+f(b)}{2},
$$

where $f: I \rightarrow \mathbb{R}$ is a convex function on $I$ and $a, b \in I, a<b$.

The Hadamard inequality provides the estimations of integral mean of a real valued function $f$. It is also considered as a base of defining a convex function, whereas convex functions lead to enrichment of the literature of the subjects like mathematical analysis, functional analysis, statistical analysis, graph theory and many other areas of mathematics. One of the celebrated fields is the theory of inequalities which is actually developed on a very fast track due to convex functions. Convex functions are also very attractive because of their graphical representation and fascinating properties and characterizations (see [26, pp. 2-15]).

(c) The Author(s) 2019. This article is distributed under the terms of the Creative Commons Attribution 4.0 International License (http://creativecommons.org/licenses/by/4.0/), which permits unrestricted use, distribution, and reproduction in any medium, provided you give appropriate credit to the original author(s) and the source, provide a link to the Creative Commons license, and indicate if changes were made. 
A finite convex function which is defined on a closed interval is bounded above by maximum of its values at the end points, but the converse needs not be true. This fact motivates us to define another class of functions, called quasi-convex functions.

Definition 2 (See, e.g., [14], p. 302) Let $I$ be an interval of real numbers. Then a function $f: I \rightarrow \mathbb{R}$ is said to be quasi-convex function, if for all $a, b \in I$ and $0 \leq t \leq 1$ the following inequality holds:

$$
f(t a+(1-t) b) \leq \max \{f(a), f(b)\} .
$$

Example $1.1([15$, p. 83$])$ The function $f:[-2,2] \rightarrow \mathbb{R}$, given by

$$
f(x)= \begin{cases}1, & x \in[-2,-1], \\ x^{2}, & x \in(-1,2]\end{cases}
$$

is not a convex function on $[-2,2]$ but it is quasi-convex function on $[-2,2]$.

Therefore it is noted that the class of quasi-convex functions contains the class of finite convex functions defined on finite closed intervals. The Hadamard and Hadamardtype inequalities for quasi-convex functions have been studied by many researchers (see $[14,15,28]$ and the references therein). For more fractional inequalities of Hadamard and Hadamard-type we suggest references $[1,2,8,10-13,21]$. Our aim is to study these inequalities in fractional calculus.

Fractional calculus is the study of classical calculus in a general form. For example integration and differentiation of arbitrary order is the key of this subject. There are a lot of integral representations of derivatives and integrations of functions of arbitrary order. For details see $[5,18-20,23,30]$. Next we give the definition of a generalized Mittag-Leffler function $E_{\mu, \alpha, l}^{\gamma, \delta, c}(\cdot ; p)$ which leads to generalized fractional integral operators. Mittag-Leffler functions are very useful in the solution of differential equations due to their natural existence in their solutions. For details we suggest $[3,4,7,16,18,19]$.

Definition 3 ([6, p. 1381, Eq. (2.8)]) Let $\mu, \alpha, l, \gamma, c \in \mathbb{C}, \Re(\mu), \Re(\alpha), \Re(l)>0, \Re(c)>\Re(\gamma)>$ 0 with $p \geq 0, \delta>0$ and $0<k \leq \delta+\Re(\mu)$. Then the extended generalized Mittag-Leffler function $E_{\mu, \alpha, l}^{\gamma, \delta, k, c}(t ; p)$ is defined by

$$
E_{\mu, \alpha, l}^{\gamma, \delta, k, c}(t ; p)=\sum_{n=0}^{\infty} \frac{\beta_{p}(\gamma+n k, c-\gamma)}{\beta(\gamma, c-\gamma)} \frac{(c)_{n k}}{\Gamma(\mu n+\alpha)} \frac{t^{n}}{(l)_{n \delta}}
$$

where $\beta_{p}$ is the generalized beta function defined by

$$
\beta_{p}(x, y)=\int_{0}^{1} t^{x-1}(1-t)^{y-1} e^{-\frac{p}{t(1-t)}} d t
$$

and $(c)_{n k}$ is the Pochhammer symbol defined as $(c)_{n k}=\frac{\Gamma(c+n k)}{\Gamma(c)}$.

In [6], properties of the generalized Mittag-Leffler function are discussed and it is given that $E_{\mu, \alpha, l}^{\gamma, \delta, c, c}(t ; p)$ is absolutely convergent for $k<\delta+\Re(\mu)$. Let $S$ be the sum of series of 
absolute terms of the Mittag-Leffler function $E_{\mu, \alpha, l}^{\gamma, \delta, k, c}(t ; p)$, then we have $\left|E_{\mu, \alpha, l}^{\gamma, \delta, k, c}(t ; p)\right| \leq S$. We use this property of the Mittag-Leffler function frequently in our results. Also in [6, p. 1384, Eqs. (2.11) and (2.12)], the derivative property of the generalized Mittag-Leffler function is discussed.

Theorem 1.2 If $m \in \mathbb{N}, \omega, \mu, \alpha, l, \gamma, c \in \mathbb{C}, \Re(\mu), \Re(\alpha), \Re(l)>0, \Re(c)>\Re(\gamma)>0$ with $p \geq 0$, $\delta>0$ and $0<k<\delta+\Re(\mu)$, then

$$
\begin{aligned}
& \left(\frac{d}{d t}\right)^{m} E_{\mu, \alpha, l}^{\gamma, \delta, k, c}(t ; p) \\
& \quad \frac{(c)_{m k}}{(l)_{m \delta}} \sum_{n=0}^{\infty} \frac{\beta_{p}(\gamma+(n+m) k, c-\gamma)}{\beta(\gamma, c-\gamma)} \frac{(c+m k)_{n k}}{\Gamma(\mu(n+m)+\alpha)} \frac{(n+1)_{m} t^{n}}{(l+m \delta)_{n \delta}}, \\
& \left(\frac{d}{d t}\right)^{m}\left[t^{\alpha-1} E_{\mu, \alpha, l}^{\gamma, \delta, k}\left(\omega t^{\mu} ; p\right)\right]=t^{\alpha-m-1} E_{\mu, \alpha-m, l}^{\gamma, \delta, c}\left(\omega t^{\mu} ; p\right), \quad \Re(\alpha)>m .
\end{aligned}
$$

Remark 1.3 (2) is a generalization of the following functions:

(i) setting $p=0$, it reduces to the Salim-Faraj function $E_{\mu, \alpha, l}^{\gamma, \delta, k, c}(t)$ defined in [27, p. 2, Eq. (6)],

(ii) setting $l=\delta=1$, it reduces to the function $E_{\mu, \alpha}^{\gamma, k, c}(t ; p)$ defined by Rahman et al. in [25, p. 4247, Eq. (2.1)],

(iii) setting $p=0$ and $l=\delta=1$, it reduces to the Shukla-Prajapati function $E_{\mu, \alpha}^{\gamma, k}(t)$ defined in [29, p. 798, Eq. (1.4)] see also [30, p. 3, Eq. (1.13)],

(iv) setting $p=0$ and $l=\delta=k=1$, it reduces to the Prabhakar function $E_{\mu, \alpha}^{\gamma}(t)$ defined in [24, p. 7, Eq. (1.3)].

The upcoming generalized fractional integral operators containing the extended generalized Mittag-Leffler function has been used to generalize several fractional integral inequalities for convex and related functions (see $[10,13,17]$ and the references therein). These operators are also useful to generalize fractional differential equations having their solutions in terms of Mittag-Leffler functions. The left-sided and right-sided generalized fractional integral operators $\epsilon_{\mu, \alpha, l, \omega, a^{+}}^{\gamma, \delta, k, c}$ and $\epsilon_{\mu, \alpha, l, \omega, b^{-}}^{\gamma, \delta, k,}$ are defined as follows:

Definition 4 ([6, p. 1385, Eq. (2.13)]) Let $\omega, \mu, \alpha, l, \gamma, c \in \mathbb{C}, \Re(\mu), \Re(\alpha), \Re(l)>0, \Re(c)>$ $\Re(\gamma)>0$ with $p \geq 0, \delta>0$ and $0<k \leq \delta+\Re(\mu)$. Let $f \in L_{1}[a, b]$ and $x \in[a, b]$. Then the generalized fractional integral operators $\epsilon_{\mu, \alpha, l, \omega, a^{+}}^{\gamma, \delta, k, c} f$ and $\epsilon_{\mu, \alpha, l, \omega, b}^{\gamma, \delta, k, c} f$ are defined by

$$
\left(\epsilon_{\mu, \alpha, l, \omega, a^{+}}^{\gamma, \delta, k, c} f\right)(x ; p)=\int_{a}^{x}(x-t)^{\alpha-1} E_{\mu, \alpha, l}^{\gamma, \delta, k, c}\left(\omega(x-t)^{\mu} ; p\right) f(t) d t
$$

and

$$
\left(\epsilon_{\mu, \alpha, l, \omega, b-b}^{\gamma, \delta, c} f\right)(x ; p)=\int_{x}^{b}(t-x)^{\alpha-1} E_{\mu, \alpha, l}^{\gamma, \delta, k, c}\left(\omega(t-x)^{\mu} ; p\right) f(t) d t
$$

In [6], several properties of the extended generalized Mittag-Leffler function and corresponding generalized fractional operators have been studied. In particular in [10, p. 3, Eqs. (5) and (6)], it is proved that

$$
\left(\epsilon_{\mu, \alpha, l, \omega, a^{+}}^{\gamma, \delta, k, c}\right)(x ; p)=(x-a)^{\alpha} E_{\mu, \alpha+1, l}^{\gamma, \delta, k}\left(w(x-a)^{\mu} ; p\right)
$$


and we denoted

$$
C_{\alpha, \omega, a^{+}}(x ; p)=\left(\epsilon_{\mu, \alpha, l, \omega, a}^{\gamma, \delta, c, c} 1\right)(x ; p) .
$$

Similarly

$$
\left(\epsilon_{\mu, \beta, l, \omega, b^{-}}^{\gamma, \delta, k}-1\right)(x ; p)=(b-x)^{\beta} E_{\mu, \beta+1, l}^{\gamma, \delta, k}\left(w(b-x)^{\mu} ; p\right)
$$

and we denoted

$$
C_{\beta, \omega, b^{-}}(x ; p)=\left(\epsilon_{\mu, \beta, l, \omega, b^{-}}^{\gamma, \delta, k}-(x ; p)\right.
$$

which we will use in our results in the sequel.

Remark 1.4 (4) and (5) are the generalizations of the following fractional integral operators:

(i) setting $p=0$, it reduces to the fractional integral operators defined by Salim-Faraj in [27, p. 2, Eq. (8)],

(ii) setting $l=\delta=1$, it reduces to the fractional integral operators defined by Rahman et al. in [25, p. 4247, Eq. (2.2)],

(iii) setting $p=0$ and $l=\delta=1$, it reduces to the fractional integral operators defined by Srivastava-Tomovski in [30, p. 5, Eq. (2.12)],

(iv) setting $p=0$ and $l=\delta=k=1$, it reduces to the fractional integral operators defined by Prabhakar in [24, p. 7, Eqs. (1.2) and (1.4)],

(v) setting $p=\omega=0$, it reduces to the left-sided and right-sided Riemann-Liouville fractional integrals.

Fractional integral inequalities are very important in the theory and applications of differential equations. Such inequalities are also of great importance in the mathematical modeling of the fractional boundary value problems. The aim of this paper is to establish an estimation of the generalized fractional integral operators (4), (5) studied in [6] by using quasi-convex functions. Actually this is a compact formula which gives estimations of all fractional integral operators comprised in Remark 1.4. Further we prove some estimations of the Hadamard-type inequality given in [17, Theorem 2.1, p. 4]. Finally, these results are applied to particular quasi-convex functions, in the result some recurrence inequalities for the generalized Mittag-Leffler functions are obtained.

We organize the results in next two sections. In Sect. 2, first we give the estimate of the sum of left-sided and right-sided generalized fractional integrals defined in (4) and (5). Then an identity is established to give the estimations of the Hadamard-type inequality [17, Theorem 2.1, p. 4]. Also we reproduce several published results as particular cases of these results. In Sect. 3, applications of these results are discussed by using some examples of quasi-convex functions.

\section{Main results}

The very first result provides the bound of the sum of the left-sided and right-sided fractional integrals via quasi-convex functions. 
Theorem 2.1 Let $f:[a, b] \rightarrow \mathbb{R}$ be a function such that $f \in L_{1}[a, b]$ with $a<b$. Iff is quasiconvex on $[a, b]$, then for generalized fractional integral operators (4) and (5) the following inequality holds:

$$
\begin{aligned}
& \left(\epsilon_{\mu, \alpha, l, \omega^{\prime}, a^{+}}^{\gamma, \delta, k, c} f\right)(b ; p)+\left(\epsilon_{\mu, \beta, l, \omega^{\prime}, b^{\prime}}^{\gamma, \delta, c} f\right)(a ; p) \\
& \quad \leq \max \{f(a), f(b)\}\left(C_{\alpha, \omega^{\prime}, a^{+}}(b ; p)+C_{\beta, \omega^{\prime}, b^{-}}(a ; p)\right),
\end{aligned}
$$

where $\omega^{\prime}=\frac{\omega}{(b-a)^{\mu}}$.

Proof Since $f$ is quasi-convex function, by using (1) one can has the following inequality:

$$
\begin{aligned}
& \int_{0}^{1} t^{\alpha-1} E_{\mu, \alpha, l}^{\gamma, \delta, k, c}\left(\omega t^{\mu} ; p\right) f(t a+(1-t) b) d t \\
& \quad \leq \max \{f(a), f(b)\} \int_{0}^{1} t^{\alpha-1} E_{\mu, \alpha, l}^{\gamma, \delta, k, c}\left(\omega t^{\mu} ; p\right) d t .
\end{aligned}
$$

Make the substitution $x=t a+(1-t) b$, that is, $t=\frac{b-x}{b-a}$ in the inequality (9). Then it takes the form

$$
\begin{aligned}
& \int_{a}^{b}\left(\frac{b-x}{b-a}\right)^{\alpha-1} E_{\mu, \alpha, l}^{\gamma, \delta, k, c}\left(\omega\left(\frac{b-x}{b-a}\right)^{\mu} ; p\right) f(x) \frac{d x}{b-a} \\
& \quad \leq \max \{f(a), f(b)\} \int_{a}^{b}\left(\frac{b-x}{b-a}\right)^{\alpha-1} E_{\mu, \alpha, l}^{\gamma, \delta, k, c}\left(\omega\left(\frac{b-x}{b-a}\right)^{\mu} ; p\right) \frac{d x}{b-a} .
\end{aligned}
$$

By using (4) of Definition 4, one can get

$$
\left(\epsilon_{\mu, \alpha, l, \omega^{\prime}, a^{+}}^{\gamma, \delta, c} f\right)(b ; p) \leq C_{\alpha, \omega^{\prime}, a^{+}}(b ; p) \max \{f(a), f(b)\} .
$$

Also by using quasi-convexity in the form $f((1-t) a+t b) \leq \max \{f(a), f(b)\}$, the following inequality can be obtained:

$$
\begin{aligned}
& \int_{0}^{1} t^{\beta-1} E_{\mu, \beta, l}^{\gamma, \delta, k, c}\left(\omega t^{\mu} ; p\right) f((1-t) a+t b) d t \\
& \quad \leq \max \{f(a), f(b)\} \int_{0}^{1} t^{\beta-1} E_{\mu, \beta, l}^{\gamma, \delta, k, c}\left(\omega t^{\mu} ; p\right) d t .
\end{aligned}
$$

Now make the substitution $y=(1-t) a+t b$, that is, $t=\frac{y-a}{b-a}$ in the inequality (12). Then it takes the form

$$
\begin{aligned}
& \int_{a}^{b}\left(\frac{y-a}{b-a}\right)^{\beta-1} E_{\mu, \beta, l}^{\gamma, \delta, k, c}\left(\omega\left(\frac{y-a}{b-a}\right)^{\mu} ; p\right) f(y) \frac{d y}{b-a} \\
& \quad \leq \max \{f(a), f(b)\} \int_{a}^{b}\left(\frac{y-a}{b-a}\right)^{\beta-1} E_{\mu, \beta, l}^{\gamma, \delta, k, c}\left(\omega\left(\frac{y-a}{b-a}\right)^{\mu} ; p\right) \frac{d y}{b-a} .
\end{aligned}
$$

By using (5) of Definition 4, one can get

$$
\left(\epsilon_{\mu, \beta, l, \omega^{\prime}, b^{-}}^{\gamma, \delta, k)} f(a ; p) \leq C_{\beta, \omega^{\prime}, b^{-}}(a ; p) \max \{f(a), f(b)\} .\right.
$$

Adding (11) and (14), we get (8), which is the required result. 
Corollary 2.2 Setting $\alpha=\beta$ in (8), then we get the following inequality:

$$
\begin{aligned}
& \left(\epsilon_{\mu, \alpha, l, \omega^{\prime}, a^{+}}^{\gamma, \delta, k, c} f\right)(b ; p)+\left(\epsilon_{\mu, \alpha, l, \omega^{\prime}, b^{-}}^{\gamma, \delta, c} f\right)(a ; p) \\
& \quad \leq \max \{f(a), f(b)\}\left(C_{\alpha, \omega^{\prime}, a^{+}}(b ; p)+C_{\alpha, \omega^{\prime}, b^{-}}(a ; p)\right) .
\end{aligned}
$$

Corollary 2.3 ([22, Theorem 2.1, p. 169]) Setting $\omega=p=0$ in (15), then we get the following inequality for the Riemann-Liouville fractional integrals:

$$
I_{a^{+}}^{\alpha} f(b)+I_{b^{-}}^{\alpha} f(a) \leq \frac{2(b-a)^{\alpha}}{\Gamma(\alpha+1)} \max \{f(a), f(b)\}
$$

Remark 2.4 If we take $\alpha=1$ in (16), then we get the following inequality for a quasi-convex function, which is related to the Hadamard inequality given by Dragomir and Pearce in $[9$, Theorem 3.3, p. 381]:

$$
\frac{1}{b-a} \int_{a}^{b} f(t) d t \leq \max \{f(a), f(b)\}
$$

The following identity is very important to give the Hadamard-type inequalities.

Lemma 2.5 Let $f:[a, b] \rightarrow \mathbb{R}$ be a function such that $f^{\prime} \in L_{1}[a, b]$ with $a<b$. Then for the generalized fractional integral operators (4) and (5) the following identity holds:

$$
\begin{aligned}
\frac{f(a)+}{2} & f(b) \\
& \times\left[\left(\epsilon_{\mu, \alpha-1, l, \omega^{\prime}, a^{+}}^{\gamma, \delta, k, c}\right)(b ; p)+\left(\epsilon_{\mu, \alpha-1, l, \omega^{\prime}, b}^{\gamma, \delta} f\right)(a ; p)\right] \\
= & \frac{b-a}{2 E_{\mu, \alpha, l}^{\gamma, \delta, c, c}(\omega ; p)} \int_{0}^{1}\left((1-t)^{\alpha-1} E_{\mu, \alpha, l}^{\gamma, \delta, k, c}\left(\omega(1-t)^{\mu} ; p\right)-t^{\alpha-1} E_{\mu, \alpha, l}^{\gamma, \delta, k, c, c}\left(\omega t^{\mu} ; p\right)\right) \\
& \times f^{\prime}(t a+(1-t) b) d t,
\end{aligned}
$$

where $\omega^{\prime}=\frac{\omega}{(b-a)^{\mu}}$.

Proof One can note that

$$
\begin{aligned}
& \frac{b-a}{2 E_{\mu, \alpha, l}^{\gamma, \delta, k, c}(\omega ; p)} \int_{0}^{1}\left((1-t)^{\alpha-1} E_{\mu, \alpha, l}^{\gamma, \delta, k, c}\left(\omega(1-t)^{\mu} ; p\right)-t^{\alpha-1} E_{\mu, \alpha, l}^{\gamma, \delta, k, c}\left(\omega t^{\mu} ; p\right)\right) \\
& \quad \times f^{\prime}(t a+(1-t) b) d t \\
& =\frac{b-a}{2 E_{\mu, \alpha, l}^{\gamma, \delta, k, c}(\omega ; p)} \int_{0}^{1}(1-t)^{\alpha-1} E_{\mu, \alpha, l}^{\gamma, \delta, k, c}\left(\omega(1-t)^{\mu} ; p\right) f^{\prime}(t a+(1-t) b) d t \\
& \quad-\frac{b-a}{2 E_{\mu, \alpha, l}^{\gamma, \delta, c}(\omega ; p)} \int_{0}^{1} t^{\alpha-1} E_{\mu, \alpha, l}^{\gamma, \delta, k, c}\left(\omega t^{\mu} ; p\right) f^{\prime}(t a+(1-t) b) d t .
\end{aligned}
$$


We first consider the first term of right hand side of (19): putting $z=1-t$, that is, $t=1-z$ and using the derivative property (3) of the Mittag-Leffler function, it takes the form

$$
\begin{aligned}
\frac{b-a}{2 E_{\mu, \alpha, l}^{\gamma, \delta, k, c}(\omega ; p)} \int_{0}^{1} z^{\alpha-1} E_{\mu, \alpha, l}^{\gamma, \delta, k, c}\left(\omega z^{\mu} ; p\right) f^{\prime}((1-z) a+z b) d z \\
=\frac{b-a}{2 E_{\mu, \alpha, l}^{\gamma, \delta, k, c}(\omega ; p)}\left[\frac{E_{\mu, \alpha, l}^{\gamma, \delta, k, c}(\omega ; p) f(b)}{b-a}\right. \\
\left.\quad-\frac{1}{b-a} \int_{0}^{1} z^{\alpha-2} E_{\mu, \alpha-1, l}^{\gamma, \delta, k, c}\left(\omega z^{\mu} ; p\right) f((1-z) a+z b) d z\right] .
\end{aligned}
$$

Making substitution $x=(1-z) a+z b$ in the above and then using (5) of Definition 4, we get

$$
\begin{aligned}
& \frac{b-a}{2 E_{\mu, \alpha, l}^{\gamma, \delta, k, c}(\omega ; p)} \int_{0}^{1} z^{\alpha-1} E_{\mu, \alpha, l}^{\gamma, \delta, k}\left(\omega z^{\mu} ; p\right) f^{\prime}((1-z) a+z b) d z \\
& =\frac{b-a}{2 E_{\mu, \alpha, l}^{\gamma, \delta, k, c}(\omega ; p)}\left[\frac{E_{\mu, \alpha, l}^{\gamma, \delta, k, c}(\omega ; p) f(b)}{b-a}-\frac{1}{(b-a)^{\alpha}}\left(\epsilon_{\mu, \alpha-1, l, \omega^{\prime}, b}^{\gamma, \delta, k, c} f\right)(a ; p)\right] .
\end{aligned}
$$

Similarly consider the second term of right hand side of (19), we get

$$
\begin{aligned}
& -\frac{b-a}{2 E_{\mu, \alpha, l}^{\gamma, \delta, k, c}(\omega ; p)} \int_{0}^{1} t^{\alpha-1} E_{\mu, \alpha, l}^{\gamma, \delta, k, c}\left(\omega t^{\mu} ; p\right) f^{\prime}(t a+(1-t) b) d t \\
& =-\frac{b-a}{2 E_{\mu, \alpha, l}^{\gamma, \delta, k, c}(\omega ; p)}\left[-\frac{E_{\mu, \alpha, l}^{\gamma, \delta, c, c}(\omega ; p) f(a)}{b-a}+\frac{1}{(b-a)^{\alpha}}\left(\epsilon_{\mu, \alpha-1, l, \omega^{\prime}, a^{+}}^{\gamma, \delta, k, c} f\right)(b ; p)\right],
\end{aligned}
$$

here we use substitution $x=t a+(1-t) b$.

Now by using the final form of both terms in (19), identity (18) is established.

In the following we give a Hadamard-type inequality by using the above lemma.

Theorem 2.6 Let $f:[a, b] \rightarrow \mathbb{R}$ be a differentiable function such that $f^{\prime} \in L_{1}[a, b]$ with $a<b$. If $\left|f^{\prime}\right|$ is quasi-convex on $[a, b]$, then for the generalized fractional integral operators (4) and (5) the following inequality holds:

$$
\begin{aligned}
& \mid \frac{f(a)+f(b)}{2}-\frac{1}{2(b-a)^{\alpha-1} E_{\mu, \alpha, l}^{\gamma, \delta, c, c}(\omega ; p)} \\
& \quad \times\left[\left(\epsilon_{\mu, \alpha-1, l, \omega^{\prime}, a^{+}}^{\gamma, \delta, k)} f\right)(b ; p)+\left(\epsilon_{\mu, \alpha-1, l, \omega^{\prime}, b}^{\gamma, \delta, k} f\right)(a ; p)\right] \mid \\
& \leq \frac{(b-a) S}{\alpha E_{\mu, \alpha, l}^{\gamma, \delta, c, c}(\omega ; p)} \max \left\{\left|f^{\prime}(a)\right|,\left|f^{\prime}(b)\right|\right\}
\end{aligned}
$$

for $k<\delta+\Re(\mu)$, where $\omega^{\prime}=\frac{\omega}{(b-a)^{\mu}}$. 
Proof Using Lemma 2.5 and the properties of the modulus, we have

$$
\begin{aligned}
& \mid \frac{f(a)+f(b)}{2}-\frac{1}{2(b-a)^{\alpha-1} E_{\mu, \alpha, l}^{\gamma, \delta, k, c}(\omega ; p)} \\
& \quad \times\left[\left(\epsilon_{\mu, \alpha-1, l, \omega^{\prime}, a^{+}}^{\gamma, \delta, k, c} f\right)(b ; p)+\left(\epsilon_{\mu, \alpha-1, l, \omega^{\prime}, b^{-}}^{\gamma, \delta, k, c} f\right)(a ; p)\right] \mid \\
& \leq \quad \frac{b-a}{2 E_{\mu, \alpha, l}^{\gamma, \delta, k, c}(\omega ; p)} \int_{0}^{1}\left|(1-t)^{\alpha-1} E_{\mu, \alpha, l}^{\gamma, \delta, k, c}\left(\omega(1-t)^{\mu} ; p\right)-t^{\alpha-1} E_{\mu, \alpha, l}^{\gamma, \delta, k, c}\left(\omega t^{\mu} ; p\right)\right| \\
& \quad \times \mid f^{\prime}(t a+(1-t) b \mid d t \\
& \leq \frac{b-a}{2 E_{\mu, \alpha, l}^{\gamma, \delta, k, c}(\omega ; p)}\left(\int_{0}^{1}\left|(1-t)^{\alpha-1} E_{\mu, \alpha, l}^{\gamma, \delta, k, c}\left(\omega(1-t)^{\mu} ; p\right)\right|\right. \\
& \left.\quad+\int_{0}^{1}\left|t^{\alpha-1} E_{\mu, \alpha, l}^{\gamma, \delta, k, c}\left(\omega t^{\mu} ; p\right)\right|\right) \mid f^{\prime}(t a+(1-t) b \mid d t .
\end{aligned}
$$

Since $\left|f^{\prime}\right|$ is quasi-convex, also using absolute convergence of the Mittag-Leffler function, we have

$$
\begin{aligned}
& \mid \frac{f(a)+f(b)}{2}-\frac{1}{2(b-a)^{\alpha-1} E_{\mu, \alpha, l}^{\gamma, \delta, k, c}(\omega ; p)} \\
& \quad \times\left[\left(\epsilon_{\mu, \alpha-1, l, \omega^{\prime}, a^{+}}^{\left.\gamma, \delta), c,(b ; p)+\left(\epsilon_{\mu, \alpha-1, l, \omega^{\prime}, b^{-}}^{\gamma, \delta, k, c} f\right)(a ; p)\right] \mid}\right.\right. \\
& \leq \frac{(b-a) S}{2 E_{\mu, \alpha, l}^{\gamma, \delta, k, c}(\omega ; p)}\left(\int_{0}^{1}\left|(1-t)^{\alpha-1}\right| d t+\int_{0}^{1}\left|t^{\alpha-1}\right| d t\right) \max \left\{\left|f^{\prime}(a)\right|,\left|f^{\prime}(b)\right|\right\} .
\end{aligned}
$$

After simple calculation we get (21), which is the required result.

Corollary 2.7 Setting $\omega=p=0$ in (21), then we get the following inequality for RiemannLiouville fractional integrals:

$$
\begin{aligned}
& \left|\frac{f(a)+f(b)}{2}-\frac{\Gamma(\alpha)}{2(b-a)^{\alpha-1}}\left[I_{a^{+}}^{\alpha-1} f(b)+I_{b^{-}}^{\alpha-1} f(a)\right]\right| \\
& \leq \frac{b-a}{\alpha} \max \left\{\left|f^{\prime}(a)\right|,\left|f^{\prime}(b)\right|\right\} .
\end{aligned}
$$

Corollary 2.8 If we take $\alpha=2$ in (24), then we get the following inequality for a quasiconvex function:

$$
\left|\frac{f(a)+f(b)}{2}-\frac{1}{b-a} \int_{a}^{b} f(t) d t\right| \leq \frac{b-a}{2} \max \left\{\left|f^{\prime}(a)\right|,\left|f^{\prime}(b)\right|\right\} .
$$

In the following we give the Hadamard-type inequality by using Lemma 2.5, Hölder's inequality and quasi-convexity of $\left|f^{\prime}\right|^{q}$.

Theorem 2.9 Let $f:[a, b] \rightarrow \mathbb{R}$ be a differentiable function such that $f^{\prime} \in L_{1}[a, b]$ with $a<b$. If $\left|f^{\prime}\right|^{q}, q>1$ is quasi-convex on $[a, b]$, then for the generalized fractional integral 
operators (4) and (5) the following inequality holds:

$$
\begin{aligned}
& \mid \frac{f(a)+f(b)}{2}-\frac{1}{2(b-a)^{\alpha-1} E_{\mu, \alpha, l}^{\gamma, \delta, k, c}(\omega ; p)} \\
& \quad \times\left[\left(\epsilon_{\mu, \alpha-1, l, \omega^{\prime}, a^{+}}^{\gamma, \delta, k, c}\right)(b ; p)+\left(\epsilon_{\mu, \alpha-1, l, \omega^{\prime}, b^{-}}^{\gamma, \delta, k, c} f\right)(a ; p)\right] \mid \\
& \leq \frac{(b-a) S}{E_{\mu, \alpha, l}^{\gamma, \delta, k, c}(\omega ; p)} \frac{1}{((\alpha-1) p+1)^{\frac{1}{p}}}\left(\max \left\{\left|f^{\prime}(a)\right|^{q},\left|f^{\prime}(b)\right|^{q}\right\}\right)^{\frac{1}{q}}
\end{aligned}
$$

for $k<\delta+\Re(\mu)$ and $\frac{1}{p}+\frac{1}{q}=1$, where $\omega^{\prime}=\frac{\omega}{(b-a)^{\mu}}$.

Proof From Lemma 2.5, properties of the modulus and Hölder's inequality, we have

$$
\begin{aligned}
\mid \frac{f(a)}{2}+f(b) & -\frac{1}{2(b-a)^{\alpha-1} E_{\mu, \alpha, l}^{\gamma, \delta, k, c}(\omega ; p)} \\
& \times\left[\left(\epsilon_{\mu, \alpha-1, l, \omega^{\prime}, a^{+}}^{\gamma, \delta} f\right)(b ; p)+\left(\epsilon_{\mu, \alpha-1, l, \omega^{\prime}, b^{-}}^{\gamma, \delta, k, c}\right)(a ; p)\right] \mid \\
\leq & \frac{b-a}{2 E_{\mu, \alpha, l}^{\gamma, \delta, k, c}(\omega ; p)} \int_{0}^{1}\left|(1-t)^{\alpha-1} E_{\mu, \alpha, l}^{\gamma, \delta, k, c}\left(\omega(1-t)^{\mu} ; p\right)-t^{\alpha-1} E_{\mu, \alpha, l}^{\gamma, \delta, k, c}\left(\omega t^{\mu} ; p\right)\right| \\
& \times \mid f^{\prime}(t a+(1-t) b \mid d t \\
\leq & \frac{b-a}{2 E_{\mu, \alpha, l}^{\gamma, \delta, k, c}(\omega ; p)}\left(\int_{0}^{1}\left|(1-t)^{\alpha-1} E_{\mu, \alpha, l}^{\gamma, \delta, k, c}\left(\omega(1-t)^{\mu} ; p\right)\right|\right. \\
& \left.+\int_{0}^{1}\left|t^{\alpha-1} E_{\mu, \alpha, l}^{\gamma, \delta, k, c}\left(\omega t^{\mu} ; p\right)\right|\right) \mid f^{\prime}(t a+(1-t) b \mid d t \\
\leq & \frac{b-a}{2 E_{\mu, \alpha, l}^{\gamma, \delta, k, c}(\omega ; p)}\left(\left(\int_{0}^{1}\left|(1-t)^{\alpha-1} E_{\mu, \alpha, l}^{\gamma, \delta, k}\left(\omega(1-t)^{\mu} ; p\right)\right|^{p} d t\right)^{\frac{1}{p}}\right. \\
& \left.+\left(\int_{0}^{1}\left|t^{\alpha-1} E_{\mu, \alpha, l}^{\gamma, \delta, k, c}\left(\omega t^{\mu} ; p\right)\right|^{p} d t\right)^{\frac{1}{p}}\right)\left(\int_{0}^{1} \mid f^{\prime}\left(t a+\left.(1-t) b\right|^{q} d t\right)^{\frac{1}{q}} .\right.
\end{aligned}
$$

Since $\left|f^{\prime}\right|^{q}$ is quasi-convex, also using absolute convergence of the Mittag-Leffler function, we have

$$
\begin{aligned}
& \mid \frac{f(a)+f(b)}{2}-\frac{1}{2(b-a)^{\alpha-1} E_{\mu, \alpha, l}^{\gamma, \delta, k, c}(\omega ; p)} \\
& \quad \times\left[\left(\epsilon_{\mu, \alpha-1, l, \omega^{\prime}, a^{+}}^{\gamma, \delta} f\right)(b ; p)+\left(\epsilon_{\mu, \alpha-1, l, \omega^{\prime}, b^{-}}^{\gamma, \delta, k, c} f\right)(a ; p)\right] \mid \\
& \leq \quad \frac{(b-a) S}{2 E_{\mu, \alpha, l}^{\gamma, \delta, k, c}(\omega ; p)}\left(\left(\int_{0}^{1}\left|(1-t)^{\alpha-1}\right|^{p} d t\right)^{\frac{1}{p}}+\left(\int_{0}^{1}\left|t^{\alpha-1}\right|^{p} d t\right)^{\frac{1}{p}}\right) \\
& \quad \times\left(\max \left\{\left|f^{\prime}(a)\right|^{q},\left|f^{\prime}(b)\right|^{q}\right)^{\frac{1}{q}} .\right.
\end{aligned}
$$

After simple calculation we get (25), which is the required result. 
Corollary 2.10 Setting $\omega=p=0$ in (25), then we get the following inequality for the Riemann-Liouville fractional integrals:

$$
\begin{gathered}
\left|\frac{f(a)+f(b)}{2}-\frac{\Gamma(\alpha)}{2(b-a)^{\alpha-1}}\left[I_{a^{+}}^{\alpha-1} f(b)+I_{b^{-}}^{\alpha-1} f(a)\right]\right| \\
\leq \frac{b-a}{((\alpha-1) p+1)^{\frac{1}{p}}}\left(\max \left\{\left|f^{\prime}(a)\right|^{q},\left|f^{\prime}(b)\right|^{q}\right\}\right)^{\frac{1}{q}} .
\end{gathered}
$$

Corollary 2.11 If we take $\alpha=2$ in (28), then we get the following inequality for the quasiconvex function:

$$
\left|\frac{f(a)+f(b)}{2}-\frac{1}{b-a} \int_{a}^{b} f(t) d t\right| \leq \frac{b-a}{(p+1)^{\frac{1}{p}}}\left(\max \left\{\left|f^{\prime}(a)\right|^{q},\left|f^{\prime}(b)\right|^{q}\right\}\right)^{\frac{1}{q}} .
$$

Theorem 2.12 Let $f:[a, b] \rightarrow \mathbb{R}$ be a differentiable function such that $f^{\prime} \in L_{1}[a, b]$ with $a<b$. If $\left|f^{\prime}\right|^{q}, q \geq 1$ is quasi-convex on $[a, b]$, then for the generalized fractional integral operators (4) and (5) the following inequality holds:

$$
\begin{aligned}
& \mid \frac{f(a)+f(b)}{2}-\frac{1}{2(b-a)^{\alpha-1} E_{\mu, \alpha, l}^{\gamma, \delta, k, c}(\omega ; p)} \\
& \quad \times\left[\left(\epsilon_{\mu, \alpha-1, l, \omega^{\prime}, a^{+}}^{\gamma, \delta, c} f\right)(b ; p)+\left(\epsilon_{\mu, \alpha-1, l, \omega^{\prime}, b^{\prime}}^{\gamma, \delta, k, c} f\right)(a ; p)\right] \mid \\
& \leq \frac{(b-a) S}{\alpha E_{\mu, \alpha, l}^{\gamma, \delta, c, c}(\omega ; p)}\left(\max \left\{\left|f^{\prime}(a)\right|^{q},\left|f^{\prime}(b)\right|^{q}\right\}\right)^{\frac{1}{q}}
\end{aligned}
$$

for $k<\delta+\Re(\mu)$, where $\omega^{\prime}=\frac{\omega}{(b-a)^{\mu}}$.

Proof From Lemma 2.5, properties of the modulus and the power mean inequality, we have

$$
\begin{aligned}
\mid \frac{f(a)}{2}+f(b) & \frac{1}{2(b-a)^{\alpha-1} E_{\mu, \alpha, l}^{\gamma, \delta, k, c}(\omega ; p)} \\
& \times\left[\left(\epsilon_{\mu, \alpha-1, l, l, \omega^{\prime}, a^{+}}^{\gamma, \delta, c} f\right)(b ; p)+\left(\epsilon_{\mu, \alpha-1, l, \omega^{\prime}, b^{-}}^{\gamma, \delta, k, c}\right)(a ; p)\right] \mid \\
\leq & \frac{b-a}{2 E_{\mu, \alpha, l}^{\gamma, \delta, k, c}(\omega ; p)} \int_{0}^{1}\left|(1-t)^{\alpha-1} E_{\mu, \alpha, l}^{\gamma, \delta, k, c}\left(\omega(1-t)^{\mu} ; p\right)-t^{\alpha-1} E_{\mu, \alpha, l}^{\gamma, \delta, k, c}\left(\omega t^{\mu} ; p\right)\right| \\
& \times \mid f^{\prime}(t a+(1-t) b \mid d t \\
\leq & \frac{b-a}{2 E_{\mu, \alpha, l}^{\gamma, \delta, k, c}(\omega ; p)}\left(\int_{0}^{1} \mid(1-t)^{\alpha-1} E_{\mu, \alpha, l}^{\gamma, \delta, k, c}\left(\omega(1-t)^{\mu} ; p\right)\right. \\
& \left.-t^{\alpha-1} E_{\mu, \alpha, l}^{\gamma, \delta, k}\left(\omega t^{\mu} ; p\right) \mid d t\right)^{1-\frac{1}{q}} \times\left(\int_{0}^{1} \mid(1-t)^{\alpha-1} E_{\mu, \alpha, l}^{\gamma, \delta, c, c}\left(\omega(1-t)^{\mu} ; p\right)\right. \\
& -t^{\alpha-1} E_{\mu, \alpha, l}^{\gamma, \delta, k, c}\left(\omega t^{\mu} ; p\right)|| f^{\prime}\left(t a+\left.(1-t) b\right|^{q} d t\right)^{\frac{1}{q}} .
\end{aligned}
$$


Since $\left|f^{\prime}\right|^{q}$ is quasi-convex, also using absolute convergence of the Mittag-Leffler function, we have

$$
\begin{aligned}
& \mid \frac{f(a)+f(b)}{2}-\frac{1}{2(b-a)^{\alpha-1} E_{\mu, \alpha, l}^{\gamma, \delta, k, c}(\omega ; p)} \\
& \quad \times\left[\left(\epsilon_{\mu, \alpha-1, l, \omega^{\prime}, a^{\prime}}^{\gamma, \delta}\right)(b ; p)+\left(\epsilon_{\mu, \alpha-1, l, \omega^{\prime}, b^{\prime}}^{\gamma, \delta} f\right)(a ; p)\right] \mid \\
& \leq \frac{(b-a) S}{2 E_{\mu, \alpha, l}^{\gamma, \delta, k, c}(\omega ; p)}\left(\int_{0}^{1}\left|(1-t)^{\alpha-1}\right| d t+\int_{0}^{1}\left|t^{\alpha-1}\right| d t\right)\left(\max \left\{\left|f^{\prime}(a)\right|^{q},\left|f^{\prime}(b)\right|^{q}\right\}\right)^{\frac{1}{q}} .
\end{aligned}
$$

After simple calculation we get (29), which is the required result.

Corollary 2.13 Setting $\omega=p=0$ in (29), then we get the following inequality for RiemannLiouville fractional integrals:

$$
\begin{aligned}
& \left|\frac{f(a)+f(b)}{2}-\frac{\Gamma(\alpha)}{2(b-a)^{\alpha-1}}\left[I_{a^{+}}^{\alpha-1} f(b)+I_{b^{-}}^{\alpha-1} f(a)\right]\right| \\
& \leq \frac{b-a}{\alpha}\left(\max \left\{\left|f^{\prime}(a)\right|^{q},\left|f^{\prime}(b)\right|^{q}\right\}\right)^{\frac{1}{q}} .
\end{aligned}
$$

Corollary 2.14 If we take $\alpha=2$ in (32), then we get the following inequality for a quasiconvex function:

$$
\left|\frac{f(a)+f(b)}{2}-\frac{1}{b-a} \int_{a}^{b} f(t) d t\right| \leq \frac{b-a}{2}\left(\max \left\{\left|f^{\prime}(a)\right|^{q},\left|f^{\prime}(b)\right|^{q}\right\}\right)^{\frac{1}{q}} .
$$

\section{Applications}

Let us consider the function $f(x)=x^{2}$. The function $f$ is convex on $[a, b]$ and $\left|f^{\prime}(x)\right|=2|x|$, which is again a convex function on $[a, b]$. Since $f$ and $\left|f^{\prime}\right|$ are convex and finite on $[a, b]$, they are quasi-convex. Results of the previous section are applied for this function and inequalities among the generalized extended Mittag-Leffler function are established.

Theorem 3.1 The Mittag-Leffler function defined in (2) satisfies the following recurrence inequality:

$$
\begin{aligned}
& 2 E_{\mu, \alpha+3, l}^{\gamma, \delta, c, c}\left(\omega^{\prime}(b-a)^{\mu} ; p\right)-E_{\mu, \alpha+2, l}^{\gamma, \delta, k, c}\left(\omega^{\prime}(b-a)^{\mu} ; p\right) \\
& \quad \leq E_{\mu, \alpha+1, l}^{\gamma, \delta, k}\left(\omega^{\prime}(b-a)^{\mu} ; p\right)\left[\frac{\max \left\{a^{2}, b^{2}\right\}-\left(a^{2}+b^{2}\right)}{2(b-a)^{2}}\right],
\end{aligned}
$$

where $\omega^{\prime}=\frac{\omega}{(b-a)^{\mu}}$.

Proof For the function $f(t)=t^{2}$ the generalized fractional integral operator is evaluated as follows:

$$
\begin{aligned}
& \left(\epsilon_{\mu, \alpha, l, c, a^{+}}^{\gamma, \delta, k, c} f\right)(x ; p) \\
& \quad=\int_{a}^{x}(x-t)^{\alpha-1} E_{\mu, \alpha, l}^{\gamma, \delta, k, c}\left(w(x-t)^{\mu} ; p\right) t^{2} d t
\end{aligned}
$$




$$
\begin{aligned}
= & \int_{a}^{x}(x-t)^{\alpha-1} \sum_{n=0}^{\infty} \frac{\beta_{p}(\gamma+n k, c-\gamma)}{\beta(\gamma, c-\gamma)} \frac{(c)_{n k}}{\Gamma(\mu n+\alpha)} \frac{\omega^{n}(x-t)^{\mu n}}{(l)_{n \delta}} t^{2} d t \\
= & \sum_{n=0}^{\infty} \frac{\beta_{p}(\gamma+n k, c-\gamma)}{\beta(\gamma, c-\gamma)} \frac{(c)_{n k}}{\Gamma(\mu n+\alpha)} \frac{\omega^{n}}{(l)_{n \delta}} \int_{a}^{x}(x-t)^{\mu n+\alpha-1} t^{2} d t \\
= & (x-a)^{\alpha}\left[a^{2} E_{\mu, \alpha+1, l}^{\gamma, \delta, k, c}\left(\omega(x-a)^{\mu} ; p\right)+2 a(x-a) E_{\mu, \alpha+2, l}^{\gamma, \delta, k, c}\left(\omega(x-a)^{\mu} ; p\right)\right. \\
& \left.+2(x-a)^{2} E_{\mu, \alpha+3, l}^{\gamma, \delta, k}\left(\omega(x-a)^{\mu} ; p\right)\right] .
\end{aligned}
$$

Similarly

$$
\begin{aligned}
\left(\epsilon_{\mu, \alpha, l, c, b-}^{\gamma, \delta, k, c} f\right)(x ; p)= & (b-x)^{\alpha}\left[b^{2} E_{\mu, \alpha+1, l}^{\gamma, \delta, k, c}\left(\omega(b-x)^{\mu} ; p\right)-2 b(b-x)\right. \\
& \left.\times E_{\mu, \alpha+2, l}^{\gamma, \delta, k, c}\left(\omega(b-x)^{\mu} ; p\right)+2(b-x)^{2} E_{\mu, \alpha+3, l}^{\gamma, \delta, k, c}\left(\omega(b-x)^{\mu} ; p\right)\right] .
\end{aligned}
$$

Using (8) of Theorem 2.1 for the function $t^{2}$, it takes the form

$$
\begin{aligned}
(b-a)^{\alpha} & {\left[a^{2} E_{\mu, \alpha+1, l}^{\gamma, \delta, k, c}\left(\omega^{\prime}(b-a)^{\mu} ; p\right)+2 a(b-a) E_{\mu, \alpha+2, l}^{\gamma, \delta, k, c}\left(\omega^{\prime}(b-a)^{\mu} ; p\right)\right.} \\
& \left.+2(b-a)^{2} E_{\mu, \alpha+3, l}^{\gamma, \delta, k, c}\left(\omega^{\prime}(b-a)^{\mu} ; p\right)\right]+(b-a)^{\beta}\left[b^{2} E_{\mu, \beta+1, l}^{\gamma, \delta, k, c}\left(\omega^{\prime}(b-a)^{\mu} ; p\right)\right. \\
& \left.-2 b(b-a) E_{\mu, \beta+2, l}^{\gamma, \delta, k}\left(\omega^{\prime}(b-a)^{\mu} ; p\right)+2(b-a)^{2} E_{\mu, \beta+3, l}^{\gamma, \delta, k, c}\left(\omega^{\prime}(b-a)^{\mu} ; p\right)\right] \\
\leq & 2 \max \left\{a^{2}, b^{2}\right\}\left((b-a)^{\alpha} E_{\mu, \alpha+1, l}^{\gamma, \delta, k, c}\left(\omega^{\prime}(b-a)^{\mu} ; p\right)\right. \\
& \left.+(b-a)^{\beta} E_{\mu, \beta+1, l}^{\gamma, \delta, k, c}\left(\omega^{\prime}(b-a)^{\mu} ; p\right)\right) .
\end{aligned}
$$

Now taking $\alpha=\beta$ in (36), then after simplification we get (33).

Theorem 3.2 The Mittag-Leffler function defined in (2) satisfies the following recurrence inequality:

$$
\begin{aligned}
& \mid \frac{a^{2}+b^{2}}{2}-\frac{1}{2 E_{\mu, \alpha, l}^{\gamma, \delta, k, c}(\omega ; p)}\left[\left(a^{2}+b^{2}\right) E_{\mu, \alpha, l}^{\gamma, \delta, k, c}\left(\omega^{\prime}(b-a)^{\mu} ; p\right)\right. \\
& \left.\quad+2(b-a)^{2}\left(2 E_{\mu, \alpha+2, l}^{\gamma, \delta, k, c}\left(\omega^{\prime}(b-a)^{\mu} ; p\right)-E_{\mu, \alpha+1, l}^{\gamma, \delta, k, c}\left(\omega^{\prime}(b-a)^{\mu} ; p\right)\right)\right] \mid \\
& \quad \leq \frac{2(b-a) S}{\alpha E_{\mu, \alpha, l}^{\gamma, \delta, k, c}(\omega ; p)} \max \{|a|,|b|\},
\end{aligned}
$$

where $\omega^{\prime}=\frac{\omega}{(b-a)^{\mu}}$.

Proof By using (34), (35) and $f(t)=t^{2},\left|f^{\prime}(t)\right|=2|t|$ in (21) of Theorem 2.6, we have

$$
\begin{aligned}
& \mid \frac{a^{2}+b^{2}}{2}-\frac{1}{2(b-a)^{\alpha-1} E_{\mu, \alpha, l}^{\gamma, \delta, k}(\omega ; p)} \\
& \quad \times\left[( b - a ) ^ { \alpha } \left((b-a)^{-1}\left(a^{2}+b^{2}\right) E_{\mu, \alpha, l}^{\gamma, \delta, c, c}\left(\omega^{\prime}(b-a)^{\mu} ; p\right)\right.\right.
\end{aligned}
$$




$$
\begin{aligned}
& \left.\left.-2(b-a) E_{\mu, \alpha+1, l}^{\gamma, \delta, k, c}\left(\omega^{\prime}(b-a)^{\mu} ; p\right)+4(b-a) E_{\mu, \alpha+2, l}^{\gamma, \delta, k, c}\left(\omega^{\prime}(b-a)^{\mu} ; p\right)\right)\right] \mid \\
\leq & \frac{(b-a) S}{\alpha E_{\mu, \alpha, l}^{\gamma, \delta, k, c}(\omega ; p)} \max \{2|a|, 2|b|\} .
\end{aligned}
$$

After simplification we get (37).

Theorem 3.3 The Mittag-Leffler function defined in (2) satisfies the following recurrence inequality:

$$
\begin{aligned}
& \mid \frac{a^{2}+b^{2}}{2}-\frac{1}{2 E_{\mu, \alpha, l}^{\gamma, \delta, k, c}(\omega ; p)}\left[\left(a^{2}+b^{2}\right) E_{\mu, \alpha, l}^{\gamma, \delta, k, c}\left(\omega^{\prime}(b-a)^{\mu} ; p\right)\right. \\
& \left.\quad+2(b-a)^{2}\left(2 E_{\mu, \alpha+2, l}^{\gamma, \delta, k, c}\left(\omega^{\prime}(b-a)^{\mu} ; p\right)-E_{\mu, \alpha+1, l}^{\gamma, \delta, k}\left(\omega^{\prime}(b-a)^{\mu} ; p\right)\right)\right] \mid \\
& \quad \leq \frac{2(b-a) S}{E_{\mu, \alpha, l}^{\gamma, \delta, k, c}(\omega ; p)} \frac{1}{((\alpha-1) p+1)^{\frac{1}{p}}}\left(\max \left\{|a|^{q},|b|^{q}\right\}\right)^{\frac{1}{q}},
\end{aligned}
$$

where $\omega^{\prime}=\frac{\omega}{(b-a)^{\mu}}$.

Proof By using (34), (35) and $f(t)=t^{2},\left|f^{\prime}(t)\right|=2|t|$ in (25) of Theorem 2.9, we have

$$
\begin{aligned}
& \mid \frac{a^{2}+b^{2}}{2}-\frac{1}{2(b-a)^{\alpha-1} E_{\mu, \alpha, l}^{\gamma, \delta, k, c}(\omega ; p)} \\
& \quad \times\left[( b - a ) ^ { \alpha } \left((b-a)^{-1}\left(a^{2}+b^{2}\right) E_{\mu, \alpha, l}^{\gamma, \delta, k, c}\left(\omega^{\prime}(b-a)^{\mu} ; p\right)\right.\right. \\
& \left.\left.\quad-2(b-a) E_{\mu, \alpha+1, l}^{\gamma, k, c}\left(\omega^{\prime}(b-a)^{\mu} ; p\right)+4(b-a) E_{\mu, \alpha+2, l}^{\gamma, \delta, c}\left(\omega^{\prime}(b-a)^{\mu} ; p\right)\right)\right] \mid \\
& \leq \frac{(b-a) S}{E_{\mu, \alpha, l}^{\gamma, \delta, k, c}(\omega ; p)} \frac{1}{((\alpha-1) p+1)^{\frac{1}{p}}}\left(\max \left\{(2|a|)^{q},(2|b|)^{q}\right\}\right)^{\frac{1}{q}} .
\end{aligned}
$$

After simplification we get (39).

Theorem 3.4 The Mittag-Leffler function defined in (2) satisfies the following recurrence inequality:

$$
\begin{aligned}
& \mid \frac{a^{2}+b^{2}}{2}-\frac{1}{2 E_{\mu, \alpha, l}^{\gamma, \delta, k, c}(\omega ; p)}\left[\left(a^{2}+b^{2}\right) E_{\mu, \alpha, l}^{\gamma, \delta, k, c}\left(\omega^{\prime}(b-a)^{\mu} ; p\right)\right. \\
& \left.\quad+2(b-a)^{2}\left(2 E_{\mu, \alpha+2, l}^{\gamma, \delta, k, c}\left(\omega^{\prime}(b-a)^{\mu} ; p\right)-E_{\mu, \alpha+1, l}^{\gamma, \delta, k, c}\left(\omega^{\prime}(b-a)^{\mu} ; p\right)\right)\right] \mid \\
& \quad \leq \frac{2(b-a) S}{\alpha E_{\mu, \alpha, l}^{\gamma, \delta, k, c}(\omega ; p)}\left(\max \left\{|a|^{q},|b|^{q}\right\}\right)^{\frac{1}{q}},
\end{aligned}
$$

where $\omega^{\prime}=\frac{\omega}{(b-a)^{\mu}}$.

Proof By using (34), (35) and $f(t)=t^{2},\left|f^{\prime}(t)\right|=2|t|$ in (29) of Theorem 2.12, we have

$$
\begin{aligned}
& \mid \frac{a^{2}+b^{2}}{2}-\frac{1}{2(b-a)^{\alpha-1} E_{\mu, \alpha, l}^{\gamma, \delta, k}(\omega ; p)} \\
& \quad \times\left[( b - a ) ^ { \alpha } \left((b-a)^{-1}\left(a^{2}+b^{2}\right) E_{\mu, \alpha, l}^{\gamma, \delta, k, c}\left(\omega^{\prime}(b-a)^{\mu} ; p\right)\right.\right.
\end{aligned}
$$




$$
\begin{aligned}
& \left.\left.-2(b-a) E_{\mu, \alpha+1, l}^{\gamma, \delta, k, c}\left(\omega^{\prime}(b-a)^{\mu} ; p\right)+4(b-a) E_{\mu, \alpha+2, l}^{\gamma, \delta, k}\left(\omega^{\prime}(b-a)^{\mu} ; p\right)\right)\right] \mid \\
\leq & \frac{2(b-a) S}{\alpha E_{\mu, \alpha, l}^{\gamma, \delta, k, c}(\omega ; p)}\left(\max \left\{|a|^{q},|b|^{q}\right\}\right)^{\frac{1}{q}} .
\end{aligned}
$$

After simplification we get (41).

Next we give the following proposition for further results.

Proposition 3.5 For the generalized fractional integral operators (4) and (5) the following identity holds:

$$
\begin{aligned}
&\left(\epsilon_{\mu, \alpha, l, \omega, a^{+}}^{\gamma, \delta, k, c} f\right)(x ; p)+\left(\epsilon_{\mu, \beta, l, \omega, b-}^{\gamma, \delta, k, c} f\right)(x ; p) \\
&= \Gamma(\rho+1)\left[(x-a)^{\alpha+\rho} E_{\mu, \alpha+\rho, c+1, l}^{\gamma, \delta, c}\left(\omega(x-a)^{\mu} ; p\right)\right. \\
&\left.+(b-x)^{\beta+\rho} E_{\mu, \beta+\rho,+1, l, l}^{\gamma, \delta, c}\left(\omega(b-x)^{\mu} ; p\right)\right] .
\end{aligned}
$$

Proof Consider the power function $f(x)=(x-a)^{\rho}, \rho \geq 1$. It is clear that $f$ is convex on finite interval $[a, b]$, therefore it is quasi-convex. So, for the function $f(t)=(t-a)^{\rho}, \rho \geq 1$ the generalized fractional integral operator (4) is evaluated as follows:

$$
\begin{aligned}
& \left(\epsilon_{\mu, \alpha, l, \omega, a^{+}}^{\gamma, \delta, k)} f(x ; p)\right. \\
& \quad=\int_{a}^{x}(x-t)^{\alpha-1} E_{\mu, \alpha, l}^{\gamma, \delta, k, c}\left(\omega(x-t)^{\mu} ; p\right)(t-a)^{\rho} d t \\
& \quad=\int_{a}^{x}(x-t)^{\alpha-1} \sum_{n=0}^{\infty} \frac{\beta_{p}(\gamma+n k, c-\gamma)}{\beta(\gamma, c-\gamma)} \frac{(c)_{n k}}{\Gamma(\mu n+\alpha)} \frac{\omega^{n}(x-t)^{\mu n}}{(l)_{n \delta}}(t-a)^{\rho} d t \\
& \quad=\sum_{n=0}^{\infty} \frac{\beta_{p}(\gamma+n k, c-\gamma)}{\beta(\gamma, c-\gamma)} \frac{(c)_{n k}}{\Gamma(\mu n+\alpha)} \frac{\omega^{n}}{(l)_{n \delta}} \int_{a}^{x}(x-t)^{\mu n+\alpha-1}(t-a)^{\rho} d t,
\end{aligned}
$$

now making the substitution $t=x-u(x-a)$, to establish

$$
\int_{a}^{x}(x-t)^{\mu n+\alpha-1}(t-a)^{\rho} d t=(x-a)^{\mu n+\alpha+\rho} \beta(\mu n+\alpha, \rho+1),
$$

therefore, (44) takes the following form:

$$
\begin{aligned}
& \left(\epsilon_{\mu, \alpha, l, \omega, a^{+}}^{\gamma, \delta, k)} f(x ; p)\right. \\
& \quad=(x-a)^{\alpha+\rho} \sum_{n=0}^{\infty} \frac{\beta_{p}(\gamma+n k, c-\gamma)}{\beta(\gamma, c-\gamma)} \frac{(c)_{n k}}{\Gamma(\mu n+\alpha)} \frac{\omega^{n}(x-a)^{\mu n}}{(l)_{n \delta}} \frac{\Gamma(\mu n+\alpha) \Gamma(\rho+1)}{\Gamma(\mu n+\alpha+\rho+1)} \\
& =\Gamma(\rho+1)(x-a)^{\alpha+\rho} E_{\mu, \alpha+\rho+1, l}^{\gamma, \delta, k, c}\left(\omega(x-a)^{\mu} ; p\right) .
\end{aligned}
$$

Now consider the function $f(x)=(b-x)^{\rho}, \rho \geq 1$ which is also convex on finite interval $[a, b]$, therefore it is quasi-convex. So, for the function $f(t)=(b-t)^{\rho}, \rho \geq 1$ the generalized fractional integral operator (5) is evaluated as follows:

$$
\begin{aligned}
& \left(\epsilon_{\mu, \beta, l, \omega, b}^{\gamma, \delta, k, c} f\right)(x ; p) \\
& \quad=\int_{x}^{b}(t-x)^{\beta-1} E_{\mu, \beta, l}^{\gamma, \delta, k, c}\left(\omega(t-x)^{\mu} ; p\right)(b-t)^{\rho} d t
\end{aligned}
$$




$$
\begin{aligned}
& =\int_{x}^{b}(t-x)^{\beta-1} \sum_{n=0}^{\infty} \frac{\beta_{p}(\gamma+n k, c-\gamma)}{\beta(\gamma, c-\gamma)} \frac{(c)_{n k}}{\Gamma(\mu n+\beta)} \frac{\omega^{n}(t-x)^{\mu n}}{(l)_{n \delta}}(b-t)^{\rho} d t \\
& =\sum_{n=0}^{\infty} \frac{\beta_{p}(\gamma+n k, c-\gamma)}{\beta(\gamma, c-\gamma)} \frac{(c)_{n k}}{\Gamma(\mu n+\beta)} \frac{\omega^{n}}{(l)_{n \delta}} \int_{x}^{b}(t-x)^{\mu n+\beta-1}(b-t)^{\rho} d t,
\end{aligned}
$$

now making the substitution $t=b-u(b-x)$, to establish

$$
\int_{x}^{b}(t-x)^{\mu n+\beta-1}(b-t)^{\rho} d t=(b-x)^{\mu n+\beta+\rho} \beta(\mu n+\beta, \rho+1),
$$

therefore, (46) takes the following form:

$$
\begin{aligned}
& \left(\epsilon_{\mu, \beta, l, \omega, b-b}^{\gamma, \delta, c, c} f\right)(x ; p) \\
& \quad=(b-x)^{\beta+\rho} \sum_{n=0}^{\infty} \frac{\beta_{p}(\gamma+n k, c-\gamma)}{\beta(\gamma, c-\gamma)} \frac{(c)_{n k}}{\Gamma(\mu n+\beta)} \frac{\omega^{n}(b-x)^{\mu n}}{(l)_{n \delta}} \frac{\Gamma(\mu n+\beta) \Gamma(\rho+1)}{\Gamma(\mu n+\beta+\rho+1)} \\
& \quad=\Gamma(\rho+1)(b-x)^{\beta+\rho} E_{\mu, \beta+\rho+1, l}^{\gamma, \delta, k, c}\left(\omega(b-x)^{\mu} ; p\right) .
\end{aligned}
$$

Adding (45) and (47), then we get required identity (43).

Theorem 3.6 The Mittag-Leffler function defined in (2) satisfies the following recurrence inequality:

$$
\begin{aligned}
& E_{\mu, \alpha+\rho+1, l}^{\gamma, \delta, k, c}\left(\omega^{\prime}(b-a)^{\mu} ; p\right)+E_{\mu, \beta+\rho+1, l}^{\gamma, \delta, k, c}\left(\omega^{\prime}(b-a)^{\mu} ; p\right) \\
& \quad \leq \frac{1}{\Gamma(\rho+1)}\left(E_{\mu, \alpha+1, l}^{\gamma, \delta, k, c}\left(\omega^{\prime}(b-a)^{\mu} ; p\right)+E_{\mu, \beta+1, l}^{\gamma, \delta, k, c}\left(\omega^{\prime}(b-a)^{\mu} ; p\right)\right),
\end{aligned}
$$

where $\omega^{\prime}=\frac{\omega}{(b-a)^{\mu}}$.

Proof By using (6), (45) and $f(x)=(x-a)^{\rho}, \rho \geq 1$ in (11), we have

$$
E_{\mu, \alpha+\rho+1, l}^{\gamma, \delta, k, c}\left(\omega^{\prime}(b-a)^{\mu} ; p\right) \leq \frac{1}{\Gamma(\rho+1)} E_{\mu, \alpha+1, l}^{\gamma, \delta, k, c}\left(\omega^{\prime}(b-a)^{\mu} ; p\right) .
$$

Now by using (7), (47) and $f(x)=(b-x)^{\rho}, \rho \geq 1$ in (14), we have

$$
E_{\mu, \beta+\rho+1, l}^{\gamma, \delta, k, c}\left(\omega^{\prime}(b-a)^{\mu} ; p\right) \leq \frac{1}{\Gamma(\rho+1)} E_{\mu, \beta+1, l}^{\gamma, \delta, k, c}\left(\omega^{\prime}(b-a)^{\mu} ; p\right) .
$$

Adding (49) and (50), then we get the required inequality (48).

\section{Acknowledgements}

We thank the editor and referees for their careful reading and valuable suggestions to make the article friendly readable. The research work of Ghulam Farid is supported by COMSATS University Islamabad.

Funding

There was no funding for the publication of this paper. 
Authors' contributions

All authors have equally made contributions to this article. All authors read and approved the final manuscript.

\section{Author details}

'Department of Mathematics, Air University, Islamabad, Pakistan. ${ }^{2}$ Department of Mathematics, COMSATS University Islamabad, Attock Campus, Pakistan. ${ }^{3}$ Department of Mathematics, University of Sargodha, Sargodha, Pakistan. ${ }^{4}$ GBPS Sherani, Hazro Attock, Pakistan.

\section{Publisher's Note}

Springer Nature remains neutral with regard to jurisdictional claims in published maps and institutional affiliations.

Received: 5 October 2018 Accepted: 8 January 2019 Published online: 18 January 2019

\section{References}

1. Abbas, G., Farid, G.: Hadamard and Fejér-Hadamard type inequalities for harmonically convex functions via generalized fractional integrals. J. Anal. 25(1), 107-119 (2017)

2. Agarwal, P.: Some inequalities involving Hadamard-type k-fractional integral operators. Math. Methods Appl. Sci. 40(11), 3882-3891 (2017)

3. Agarwal, P., Al-Mdallal, Q., Cho, Y.J., Jain, S.: Fractional differential equations for the generalized Mittag-Leffler function. Adv. Differ. Equ. 2018, 58 (2018)

4. Agarwal, P., El-Sayed, A.A.: Non-standard finite difference and Chebyshev collocation methods for solving fractional diffusion equation. Phys. A, Stat. Mech. Appl. 500, 40-49 (2018)

5. Anastassiou, G.A.: Advanced Inequalities. World Scientific, Singapore (2011)

6. Andrić, M., Farid, G., Pečarić, J.: A further extension of Mittag-Leffler function. Fract. Calc. Appl. Anal. 21(5), 1377-1395 (2018)

7. Baltaeva, U., Agarwal, P.: Boundary value problems for the third order loaded equation with non characteristic type change boundaries. Math. Methods Appl. Sci. 41(9), 3307-3315 (2018)

8. Choi, J., Agarwal, P.: Certain fractional integral inequalities involving hypergeometric operators. East Asian Math. J. 30(3), 283-291 (2014)

9. Dragomir, S.S., Pearce, C.E.M.: Quasi-convex functions and Hadamard's inequality. Bull. Aust. Math. Soc. 57, 377-385 (1998)

10. Farid, G., Khan, K.A., Latif, N., Rehman, A.U., Mehmood, S.: General fractional integral inequalities for convex and m-convex functions via an extended generalized Mittag-Leffler function. J. Inequal. Appl. 2018(2018), 243 (2018)

11. Farid, G., Rehman, A.U., Zahra, M.: On Hadamard inequalities for k-fractional integrals. Nonlinear Funct. Anal. Appl. 21(3), 463-478 (2016)

12. Farid, G., Rehman, A.U., Zahra, M.: On Hadamard inequalities for relative convex function via fractional integrals. Nonlinear Anal. Forum 21(1), 77-86 (2016)

13. Farid, G., Rehman, A.Ur., Mehmood, S.: Hadamard and Fejér-Hadamard type integral inequalities for harmonically convex functions via an extended generalized Mittag-Leffler function. J. Math. Comput. Sci. 8(5), 630-643 (2018)

14. Hussain, R., Ali, A., Latif, A., Gulshan, G.: Some k-fractional associates of Hermite-Hadamard's inequality for quasi-convex functions and applications to special means. Fract. Differ. Calc. 7(2), 301-309 (2017)

15. Ion, D.A.: Some estimates on the Hermite-Hadamard inequality through quasi-convex functions. An. Univ. Craiova, Ser. Mat. Inform. 34, 82-87 (2007)

16. Jain, S., Agarwal, P., Kilicman, A.: Pathway fractional integral operator associated with 3m-parametric Mittag-Leffler functions. Int. J. Appl. Comput. Math. 4, 115 (2018)

17. Kang, S.M., Farid, G., Nazeer, W., Tariq, B.: Hadamard and Fejér-Hadamard inequalities for extended generalized fractional integrals involving special functions. J. Inequal. Appl. 2018(2018), 119 (2018)

18. Kilbas, A.A., Srivastava, H.M., Trujillo, J.J.: Theory and Applications of Fractional Differential Equations. North-Holland Mathematics Studies, vol. 204. Elsevier, New York (2006)

19. Lazarević, M.: Advanced Topics on Applications of Fractional Calculus on Control Problems, System Stability and Modeling. WSEAS Press (2014)

20. Letnikov, A.V.: Theory of differentiation with an arbitrary index. Mat. Sb. 3, 1-66 (1868) (in Russian)

21. Mehrez, K., Agarwal, P.: New Hermite-Hadamard type integral inequalities for convex functions and their applications. J. Comput. Appl. Math. 350, 274-285 (2019)

22. Özdemir, M.E., Yildiz, C.: The Hadamard's inequality for quasi-convex functions via fractional integrals. In: Annals of the University of Craiova. Mathematics and Computer Science Series, vol. 40, pp. 167-173 (2013)

23. Podlubni, I.: Fractional Differential Equations. Academic Press, San Diego (1999)

24. Prabhakar, T.R.: A singular integral equation with a generalized Mittag-Leffler function in the kernel. Yokohama Math. J. 19, 7-15 (1971)

25. Rahman, G., Baleanu, D., Qurashi, M.A., Purohit, S.D., Mubeen, S., Arshad, M.: The extended Mittag-Leffler function via fractional calculus. J. Nonlinear Sci. Appl. 10, 4244-4253 (2017)

26. Roberts, A.W., Varberg, D.E.: Convex Functions. Academic Press, New York (1973)

27. Salim, T.O., Faraj, A.W.: A generalization of Mittag-Leffler function and integral operator associated with integral calculus. J. Fract. Calc. Appl. 3(5), 1-13 (2012)

28. Set, E., Celik, B.: Fractional Hermite-Hadamard type inequalities for quasi-convex functions. Ordu Univ. J. Sci. Tech 6(1), 137-149 (2016)

29. Shukla, A.K., Prajapati, J.C.: On a generalization of Mittag-Leffler function and its properties. J. Math. Anal. Appl. 336, 797-811 (2007)

30. Srivastava, H.M., Tomovski, Z.: Fractional calculus with an integral operator containing generalized Mittag-Leffler function in the kernel. Appl. Math. Comput. 211(1), 198-210 (2009) 